\title{
Critical role of calpain in inflammation (Review)
}

\author{
JINGJING JI ${ }^{1,2}$, LEI SU ${ }^{1,3}$ and ZHIFENG LIU ${ }^{1,3}$ \\ ${ }^{1}$ Department of Critical Care Medicine, General Hospital of Guangzhou Military Command, \\ Guangzhou, Guangdong 510010; ${ }^{2}$ Graduate School, Guangzhou Medical University, Guangzhou, \\ Guangdong 510010; ${ }^{3}$ Key Laboratory of Hot Zone Trauma Care and Tissue Repair of PLA, General \\ Hospital of Guangzhou Military Command, Guangzhou, Guangdong 510010, P.R. China
}

Received October 10, 2015; Accepted September 14, 2016

DOI: $10.3892 /$ br.2016.785

\begin{abstract}
Calpains are a family of cysteine proteases, implicated in a wide range of cellular calcium-regulated functions. Evidence from previous studies using an inhibitor of calpain indicates that calpain activation is involved in the process of numerous inflammation-associated diseases. As a result of in-depth studies, calpains have been proposed to influence the process of inflammation via a variety of mechanisms. The aim of the present study is to provide an overview of recent reports regarding the role of calpain in the process of inflammation, including regulation of immune cell migration, modulation of the activation of inflammatory mediators, degradation of certain associated proteins and induction of cell apoptosis. Understanding these mechanisms may contribute to the investigation of novel therapeutic targets for inflammation-associated diseases.
\end{abstract}

\section{Contents}

1. Introduction

2. Calpains and inflammatory cells

3. Calpains and inflammatory mediators

4. Calpains and inflammation-associated diseases

5. Conclusion

\section{Introduction}

Calpains are calcium-dependent cysteine proteases, ubiquitously expressed in mammals. The classical calpains exist as heterodimers comprised of a large catalytic subunit of $80 \mathrm{kD}$ and a small regulatory subunit of $30 \mathrm{kD}$ (1). Calpain-1

Correspondence to: Dr Zhifeng Liu, Department of Critical Care Medicine, General Hospital of Guangzhou Military Command, Guangzhou, Guangdong 510010, P.R. China

E-mail: zhifengliu7797@163.com

Key words: calpain, inflammation, immune cell, inflammatory mediator, sepsis, diabetes and calpain-2 are the regularly evaluated members of this family due to their ubiquitous expression, whereas the tissue distribution of other calpain family members is limited. In the current review, the term calpain or calpains predominantly refer to calpain-1 and calpain-2 isoforms. In addition, they are termed $\mu$-calpain and m-calpain based on their requirement for a concentration of intracellular $\mathrm{Ca}^{2+}$ for activation, in the micromolar and millimolar ranges, respectively. Therefore, calpain-1 participates in more physical processes than calpain-2, due to the lower requirement of $\mathrm{Ca}^{2+}$ concentration, which is easy to achieve in vivo. The elevation of $\mathrm{Ca}^{2+}$ concentration is the primary activator of calpain, and certain other factors may activate calpain via other signaling pathways, such as the extracellular signal-regulated kinases/mitogen-activated protein kinases signaling pathway (2). The inhibition of calpain is predominantly induced by its endogenous inhibitor, calpastatin, which is responsible for the limited proteolysis of substrates. A wide variety of proteins have been considered as the substrates of calpain, including cytoskeletal and plasma membrane-associated proteins, signal transduction proteins, and transcription factors (3), indicating that calpains are significant in a wide range of cellular calcium-regulated functions.

Inflammation, a defensive reaction in living tissues and the vascular system to injurious stimuli, is essential to maintain homeostasis during infection and injury. A typical inflammatory response consists of four components as follows: inflammatory inducers, the inflammatory cells that detect them, inflammatory mediators and the target tissues that are affected by the inflammatory mediators (4). Endogenous or exogenous damage agents may activate the inflammatory cells via the pathogen-associated molecular patters or danger/damage-associated molecular patters, which induce the production of inflammatory mediators. These inflammatory mediators subsequently act on target tissues, which may activate the inflammatory cells and, in turn, amplify the inflammatory response.

Evidence from previous studies that used an inhibitor of calpain propose that the activities of calpain are involved in the process of various inflammation-associated diseases. In the majority of cases, the calpain inhibitor exerts a protective role in the inflammation-associated disease $(5,6)$. It has been established that calpain may be involved in the degradation of $\mathrm{I} \kappa \mathrm{B}$, the endogenous inhibitor of nuclear factor $(\mathrm{NF})-\kappa \mathrm{B}$, 
contributing to the activation of $\mathrm{NF}-\kappa \mathrm{B}$, a transcriptional regulatory protein, which is central to regulating the transcription of numerous inflammatory mediators, such as tumor necrosis factor (TNF)- $\alpha$, interleukin (IL)-1 and cyclooxygenase-2, promoting the process of inflammation in numerous types of disease $(7,8)$. In addition to this signaling pathway, as research continues, increasing evidence indicates that calpain may be involved in the process of inflammation via various underlying mechanisms, which are correlated with inflammatory cells and mediators.

\section{Calpains and inflammatory cells}

Macrophages. Macrophages are critical during the inflammatory process in phagocytosis and the clearance of pathogens. In resting macrophages, calpain-mediated cleavage of selenoprotein $\mathrm{K}$, between $\mathrm{Arg}^{81}$ and Gly ${ }^{82}$, is essential to maintain the inactive condition of macrophages. Macrophages are activated when cleavage is inhibited by the upregulation of the endogenous inhibitor of calpain, calpastatin, which is induced by Toll-like receptors (9).

Macrophages act as key components in the innate immune system. However, they are the main targets for certain pathogens to avoid host defenses at the same time. Group B streptococcus (GBS) induces macrophage membrane permeability disruption for the cleavage of cytoskeleton proteins by calpain (10). In addition, calpain may degrade the pro-apoptotic factors, BCL2 associated $\mathrm{X}$, apoptosis regulator and $\mathrm{BH} 3$ interacting domain death agonist, resulting in the apoptosis of macrophages under the stimuli of GBS (11). Streptococcus pyogenes-induced oncosis of macrophages is associated with calpain, which may mediate the mitochondrial permeability transition (12). Furthermore, calpain activation is involved in endoplasmic reticulum stress-mediated apoptosis during the pathogenesis of Mycobacterium kansasii (13). Leishmania infection may activate calpain-1 via the G-protein signaling pathway; the activated calpain-1 may inhibit the transcription of RNA polymerase III, impairing the function of macrophages, thus helping the pathogen to establish an infection in the host cells (14).

Neutrophils. Neutrophils, key participants in the innate system that increase significantly during inflammation, contribute to clearing pathogens by phagocytosis, as well as via other mechanisms. It has been revealed that calpain and calpastatin are critical for the chemotaxis of neutrophils. In contrast to all other cell types, constitutive calpain activity exerts a negative role in polarization and migration in resting neutrophils by regulating the activities of cell division control protein 42 homolog and Rho GTPase binding protein Rac1 (15). Inhibition of calpain activity may promote neutrophil migration associated with the activation of distinct signaling molecules (16).

Following migration to the endothelium, the morphology of neutrophils dramatically changes from spherical to flattened, which may attenuate the shear stress of blood flow, preventing cell rolling. It has been shown that this shape change is triggered by elevated physiological $\mathrm{Ca}^{2+}$ (the activator of calpain) influx, which is induced by inositol 1,4,5-triphosphate. Calpain may cleave the 4.1/ezrin/radixin/moesin domain and an actin-binding domain on the cytoskeleton. This enables the wrinkled membrane to flatten out, facilitating leukocyte plasma membrane expansion $(17,18)$.

It has been shown that calpains participate in the apoptosis of neutrophils, which usually survive for only $24 \mathrm{~h}$ on average. With the depleted endogenous inhibitor, calpastatin, the enhanced activation of calpain is sufficient to cause apoptosis of neutrophils (19). In addition to apoptosis, calpains participate in the process of apoptotic-to-necrotic transition of neutrophils, as calpain activity may prevent the ingestion of neutrophils, resulting in the release of pathogenic molecules from the death neutrophils, such as protease and hydrolases, that may damage the local tissue (20).

Lymphocytes. The expression of calpain is particularly low in resting lymphocytes; however, it has been shown that synthesis and secretion of calpain occurs in active lymphocytes, particularly in T cells (21). Inhibition of calpain activity has indicated attenuation of $\mathrm{T}$ cell proliferation, suggesting that calpain is involved in the regulation of lymphocyte proliferation (22). In addition, calpain may be implicated in the differentiation of $\mathrm{T}$ cells, as the calpain:calpastatin ratio varied during $\mathrm{T}$ helper (Th)1, Th2 and Th17 development (23). As with macrophages and neutrophils, the migration of lymphocytes may be regulated by the activation of calpain. The regulation process might be associated with an integrin on the lymphocyte surface, termed lymphocyte function-associated antigen-1 (LFA-1). In a previous study, calpain promoted LFA-1 adhesion to intercellular adhesion molecule -1 (ICAM-1), causing the T cells to remain in the lymph node or the infection area (24). Recently, however, a novel study showed that calpain-2 may contribute to turnover of LFA-1 adhesion on migrating T cells (25), which may be essential to maintaining the active state of $\mathrm{T}$ cells in peripheral blood. In addition, calpain in endothelia is essential to lymphocyte transendothelial migration (26). Furthermore, inhibition of calpain may reduce the Th1/Th17 inflammatory cytokines, including IL-12, IL-17, TNF- $\alpha$ and granulocyte colony-stimulating factor (22).

A study on the expression of calpain-1 and calpain-2 in peripheral blood lymphocytes from different age groups showed that the expression levels of calpains were decreased in the elderly group (27). As mentioned above, calpains participate in the process of proliferation, differentiation and migration. Thus, decreased expression levels of calpain may lead to inefficient immune function, which may be an explanation for the hypoimmunity of -the elderly.

\section{Calpains and inflammatory mediators}

IL-1 is known to be involved in various biological activities during the process of inflammation. In lipopolysaccharide (LPS)-induced inflammation, calpain may mediate the cleavage of pre-IL-1 $\alpha$, increasing its affinity for IL-1 receptor 1 (28). Similarly, this process occurs during necrosis-induced sterile inflammation (29). In addition, calpain activation is essential to IL-1 $\alpha$ nuclear localization, which may contribute to amplifying the inflammatory response (30). In the IL family, in addition to IL-1, calpain activity promotes the release of other cytokines, as IL-6, IL-12 and IL-17 may be suppressed by the inhibition of calpain $(22,23)$. IL-13 may activate calpain via enhancement of endoplasmic reticulum stress (31). Furthermore, TNF- $\alpha$ 
may activate cytosolic calpain-2, which may activate $\mathrm{NF}-\kappa \mathrm{B}$ activation by degrading $\mathrm{I} \kappa \mathrm{B}(32)$. In turn, $N F-\kappa \mathrm{B}$ promotes the transcription of TNF- $\alpha$ and other cytokines, thus amplifying the inflammation response.

It has been demonstrated that HSP90, a class of molecular chaperone, is a substrate of calpain. Calpains may influence the activation of various proteins, which could form complexes with HSP90 by degrading the chaperone. For example, the degradation of HSP90 decreases the level of endothelial nitric oxide synthase (eNOS), resulting in the dysfunction of vascular endothelia (33). Since HSP90 is essential to maintaining the function of the hormone binding domain, the effect of calpain on HSP90 cleavage leads to the reduced activity of glucocorticoids (34). In septic mice, calpain induces myocardial caspase-3 activation and apoptosis by diminishing Hsp90-Akt binding (35).

Calpain may regulate the interaction between inflammatory cells by modulation of the levels of adhesion molecules, such as ICAM, vascular cell adhesion molecule and monocyte chemoattractant protein-1 $(36,37)$. In addition to these molecules, calpain has been considered as a key regulator of integrin-mediated functions, as various integrin-binding partners have been identified as substrates of calpain, such as talin, kindlin and paxillin. The cleavage of talin and kindlin reduce cell adhesion and increase focal adhesion turnover, promoting the migration of cells $(38,39)$. By contrast, the cleavage of paxillin promotes cell adhesion and inhibits cell migration (40).

Nitric oxide (NO), a short-life molecule that is produced by a variety of cells, is an important regular of inflammation due to its effect on vasodilatation. Calpain activation may regulate the level of NO by regulating the level of NOS, which catalyzes the formation of NO from arginine. In addition, calpain may positively regulate the synthesis of NO by upregulation of eNOS (41). By contrast, in the majority of cases, calpain exerts a negative effect on the synthesis of NO. Due to the stimulation of glycation of low-density lipoprotein or hyperglycemia, for example, activated calpain may decrease the level of NO by downregulating the level of eNOS $(42,43)$.

\section{Calpains and inflammation-associated diseases}

Resolving inflammation. Traditional inflammation, caused by infection or injury, consequently results in resolution, due to restoration of the structure and function of affected tissues (44). Infection and injury may activate calpains in these types of resolving inflammation. The Shigella virulence effector, VirA may stimulate calpain activation by promoting $\mathrm{Ca}^{2+}$ influx. The VirA-induced calpain activity may promote the polymerization events of actin, a cytoskeletal protein, driving bacterial entry. However, in capn4 (the encoding gene of calpain)-deficient cells, there was increased proliferation of bacteria, indicating that calpain performs a dual function during infection. Calpain activation may ultimately lead to necrotic cell death by degradation of p53, thereby restricting Shigella intracellular proliferation (45). This may be a protective mechanism of the host to defend against infection. By contrast, calpain activity is involved in the apoptotic cell death of lung epithelia and aggravates the dysfunction of the epithelial barrier following Acinetobacter baumannii infection (46). An in vitro study has shown that coxsackievirus B3 (CVB3)-induced calpain activation may enhance autophagy in the early phase of infection in $\mathrm{H} 9 \mathrm{c} 2$ cardiomyocytes, and this process may be beneficial to the replication of CVB3 in the host cells (47). In addition to infection, injury (such as stretching from mechanical ventilation) may induce the activation of calpain. The mechanical stretch-activated calpain was identified as an upstream regulator in ventilator-induced lung injury. It may upregulate NOS-3 activity, inducing ICAM-1 phosphorylation and polymorphonuclear neutrophil infiltration (48).

Increased expression of calpain-1 was observed in the cardiomyocytes obtained from mice with severe sepsis (49). Calpain-1 activity may be induced by the stimulus of LPS via the activation of nicotinamide adenine dinucleotide phosphate (NADPH) oxidase (50). The rapid cleavage of dystrophin by calpain disrupts the dystrophin-glycoprotein complex, aggravating the damage to the myocardium (49). The sarcomere may be disrupted by calpain and subsequently degraded by the ubiquitin-proteasome system, resulting in cardiac contractile dysfunction and skeletal muscle wasting $(49,51)$. The activated calpain may induce the activation of caspase-3, leading to the apoptosis of cardiomyocytes (35) and pulmonary microvascular endothelial cells (52). Similarly, the calpain-induced caspase-3 signaling pathway also contributes to dysfunction of the diaphragm (53) and skeletal muscles (54). In addition to the dysfunction of organs, calpains are associated with the process of coagulation during sepsis by inducing the apoptosis of platelets (55) and promoting the release of the procoagulant microparticle (56). The above-mentioned findings imply that calpain participates in the pathogenesis of sepsis and may present as a therapeutic target in future.

Although the majority of cases indicate that the activation of calpain may promote tissue damage and that the inhibition of calpain may be beneficial, it should be noted that contradictory results have also been reported. Using an acute bacterial peritonitis model, Kumar et al (57) showed that calpain may serve a protective role during acute bacterial infection. Compared with the wide type mice, the capns1 knockout mice, in which the expression of calpain was deficient, showed impaired bacterial killing for the attenuation of IL-1 $\alpha$ release and neutrophil recruitment, suggesting that calpains may be beneficial to bacteria clearance (57). The various roles of calpain may correlate with its differential involvement in different disease states, the variation of each isoform in the calpain family and the specificity of tissues.

Non-resolving inflammation. Non-resolving inflammation, including diabetes, atherosclerosis, multiple sclerosis and certain types of cancer, is different from resolving inflammation due to the failure to recover from these types of diseases. The process of these diseases seems to be chronic from the outset without any signs of infection, however, with obvious signs of inflammation evidenced by the infiltration of the tissue by inflammatory cells (44). Studies have shown that activation of calpains is enhanced in these types of disease, suggesting that calpain may be involved in the pathogenesis. For example, evidence from the comparison of spinal cord tissue samples from Parkinson's disease patients and age-matched healthy subjects revealed that the upregulation of calpain may be associated with inflammatory response-mediated neuronal 
death for the elevation levels of inflammatory mediators and calpains (58).

Under the stimulation of hyperglycemia, the activity of calpain may be upregulated by increased $\mathrm{Na}^{+} / \mathrm{H}^{+}$exchange and the protein kinase $\mathrm{C}(\mathrm{PKC})$ signaling pathway $(43,59)$, leading to the alteration of vascular reactivity. Activated calpain may cleave the $\mathrm{Ca}^{2+}$-sensing receptor (CaSR), a $\mathrm{G}$ protein-coupled receptor, in the endothelia and smooth muscles, resulting in CaSR function loss and late complications in macrovascular disease (60). In addition, calpain may dissociate HSP90 from eNOS in the endothelia, leading to downregulation of the expression level of NO, which may increase reactive oxygen species production, thus aggravating endothelial dysfunction $(43,61)$. In chronic hyperglycemia with insulin deficiency rats, it was shown that $\mathrm{PKC}$-induced calpain-1 activation may upregulate ICAM-1 (59). The decreased NO level and increased level of adhesion molecules may contribute to endothelial dysfunction, promoting the inflammatory response in the microenviroment resulting from diabetes. In addition to microvascular dysfunction, calpains participate in the pathogenesis of diabetic cardiomyopathy under the stimulus of high glucose via the NADPH oxidase-dependent signaling pathway (62). Rac1, an essential subunit of NADPH oxidase, may be significant in the activation of calpain (63). The activated calpain-1 may induce the apoptosis of cardiomyocytes by downregulating $\mathrm{Na}^{+} / \mathrm{K}^{+}$ATPase, contributing to the cardiac complications associated with diabetes (64).

Angiotensin II may activate calpains via the activation of angiotensin II type 1 receptor signaling (65) and the upregulation of epidermal growth factor receptor (37). The activated calpains may enhance the expression of inflammatory mediators by promoting the activation of $\mathrm{NF}-\kappa \mathrm{B}$, and may increase the leukocyte-endothelium interaction and albumin permeability (65). These effects in the microcirculation may be involved in the process of cardiovascular remodeling. Similarly, certain reports have shown that calpain-1 and calpain-2 may be involved in left ventricular (LV) remodeling. A mouse model with cardiomyocyte-specific deletion of Capn4 demonstrated relatively improved cardiac function and reduced mortality following myocardial infarction (66). These results demonstrate the possible associations between the inhibition of $\mathrm{NF}-\kappa \mathrm{B}$ activation, decreased apoptosis of cardiomyocytes (66) and reduced myocardial hypertrophy and fibrosis, leading to the significant attenuation of $\mathrm{LV}$ remodeling (67).

Although many of the mechanisms of calpain activity remain unclear, it has been identified that calpains are involved in numerous types of non-resolving inflammation disease, including asthma, atherosclerosis, multiple sclerosis and rheumatoid arthritis $(22,68-70)$. These diseases make up a high proportion of the disease spectrum, however, are associated with a low possibility of complete recovery. Calpains may present as a therapeutic target for their significant activity during the pathological process of these types of chronic diseases.

\section{Conclusion}

Evidences from previous studies that used the inhibitor of calpain indicate that calpain activity contributes to the process of numerous inflammation-associated diseases. And in the majority of cases, the calpain inhibitor serves a protective role in the disease. It has been concluded that calpain is involved in the process of inflammation via a variety of mechanisms, including regulation of macrophages, neutrophils, lymphocyte migration and apoptosis, and modulation of the activation of inflammatory mediators, degradation of certain associated proteins and induction of cell apoptosis. Furthermore, there is evidence to indicate that calpain performs a protective role in the process of various types of disease $(45,57,71)$. Many of the underlying mechanisms of calpain activity and whether the effects of calpain activity influence each other remain largely unknown; therefore further investigations are required. The findings of future studies may contribute to establishing novel therapeutic targets for the treatment of inflammation-associated diseases.

\section{Acknowledgements}

The present study was supported by grants from the National Natural Science Foundation of China (grant nos. 81071529, 81101467 and 81101822), the Project Team of Natural Science Foundation of Guangdong Province (grant no. s2013030013217), the Project of Medical Research of PLA (grant no. BWS12J108) and the Guangdong Province Science and Technology Planning Project of China (grant no. 2102B031800416)

\section{References}

1. Saez ME, Ramirez-Lorca R, Moron FJ and Ruiz A: The therapeutic potential of the calpain family: New aspects. Drug Discov Today 11: 917-923, 2006.

2. Perrin BJ and Huttenlocher A: Calpain. Int J Biochem Cell Biol 34: 722-725, 2002.

3. Goll DE, Thompson VF, Li H, Wei W and Cong J: The calpain system. Physiol Rev 83: 731-801, 2003.

4. Medzhitov R: Inflammation 2010: New adventures of an old flame. Cell 140: 771-776, 2010.

5. Cuzzocrea S, McDonald MC, Mazzon E, Siriwardena D, Serraino I, Dugo L, Britti D, Mazzullo G, Caputi AP and Thiemermann C: Calpain inhibitor I reduces the development of acute and chronic inflammation. Am J Pathol 157: 2065-2079, 2000.

6. Ruetten $\mathrm{H}$ and Thiemermann C: Effect of calpain inhibitor I, an inhibitor of the proteolysis of I kappa B, on the circulatory failure and multiple organ dysfunction caused by endotoxin in the rat. Br J Pharmacol 121: 695-704, 1997.

7. Shumway SD, Maki M and Miyamoto S: The PEST domain of IkappaBalpha is necessary and sufficient for in vitro degradation by mu-calpain. J Biol Chem 274: 30874-30881, 1999.

8. Sun Z and Andersson R: NF-kappaB activation and inhibition: A review. Shock 18: 99-106, 2002.

9. Huang Z, Hoffmann FW, Norton RL, Hashimoto AC and Hoffmann PR: Selenoprotein K is a novel target of m-calpain, and cleavage is regulated by Toll-like receptor-induced calpastatin in macrophages. J Biol Chem 286: 34830-34838, 2011.

10. Fettucciari K, Quotadamo F, Noce R, Palumbo C, Modesti A, Rosati E, Mannucci R, Bartoli A and Marconi P: Group B Streptococcus (GBS) disrupts by calpain activation the actin and microtubule cytoskeleton of macrophages. Cell Microbiol 13: 859-884, 2011.

11. Fettucciari K, Fetriconi I, Mannucci R, Nicoletti I, Bartoli A, Coaccioli S and Marconi P: Group B Streptococcus induces macrophage apoptosis by calpain activation. J Immunol 176: 7542-7556, 2006.

12. Goldmann O, Sastalla I, Wos-Oxley M, Rohde M and Medina E: Streptococcus pyogenes induces oncosis in macrophages through the activation of an inflammatory programmed cell death pathway. Cell Microbiol 11: 138-155, 2009. 
13. Lim YJ, Choi HH, Choi JA, Jeong JA, Cho SN, Lee JH, Park JB, Kim HJ and Song CH: Mycobacterium kansasii-induced death of murine macrophages involves endoplasmic reticulum stress responses mediated by reactive oxygen species generation or calpain activation. Apoptosis 18: 150-159, 2013.

14. Rana T, Misra S, Mittal MK, Farrow AL, Wilson KT, Linton MF, Fazio S, Willis IM and Chaudhuri G: Mechanism of down-regulation of RNA polymerase III-transcribed non-coding RNA genes in macrophages by Leishmania. J Biol Chem 286: 6614-6626, 2011

15. Lokuta MA, Nuzzi PA and Huttenlocher A: Calpain regulates neutrophil chemotaxis. Proc Natl Acad Sci USA 100: 4006-4011, 2003.

16. Katsube M, Kato T, Kitagawa M, Noma H, Fujita $H$ and Kitagawa S: Calpain-mediated regulation of the distinct signaling pathways and cell migration in human neutrophils. J Leukoc Biol 84: 255-263, 2008.

17. Dewitt S, Francis RJ and Hallett $\mathrm{MB}: \mathrm{Ca}^{2+}$ and calpain control membrane expansion during the rapid cell spreading of neutrophils. J. Cell Sci 126, 4627-4635, 2013.

18. Dewitt S and Hallett M: Leukocyte membrane 'expansion': A central mechanism for leukocyte extravasation. J Leukoc Biol 81: 1160-1164, 2007.

19. Squier MK, Sehnert AJ, Sellins KS, Malkinson AM, Takano E and Cohen JJ: Calpain and calpastatin regulate neutrophil apoptosis. J Cell Physiol 178: 311-319, 1999

20. Francis RJ, Kotecha S and Hallett MB: $\mathrm{Ca}^{2+}$ activation of cytosolic calpain induces the transition from apoptosis to necrosis in neutrophils with externalized phosphatidylserine. J Leukoc Biol 93: 95-100, 2013.

21. Deshpande RV, Goust JM, Chakrabarti AK, Barbosa E, Hogan EL and Banik NL: Calpain expression in lymphoid cells. Increased mRNA and protein levels after cell activation. J Biol Chem 270: 2497-2505, 1995.

22. Smith AW, Doonan BP, Tyor WR, Abou-Fayssal N, Haque A and Banik NL: Regulation of Th1/Th17 cytokines and IDO gene expression by inhibition of calpain in PBMCs from MS patients. J Neuroimmunol 232: 179-185, 2011.

23. Iguchi-Hashimoto M, Usui T, Yoshifuji H, Shimizu M, Kobayashi S, Ito Y, Murakami K, Shiomi A, Yukawa N, Kawabata D, et al: Overexpression of a minimal domain of calpastatin suppresses IL-6 production and Th17 development via reduced NF- $\kappa$ B and increased STAT5 signals. PLoS One 6 : e27020, 2011.

24. Stewart MP, McDowall A and Hogg N: LFA-1-mediated adhesion is regulated by cytoskeletal restraint and by a $\mathrm{Ca}^{2+}$-dependent protease, calpain. J Cell Biol 140: 699-707, 1998.

25. Svensson L, McDowall A, Giles KM, Stanley P, Feske S and Hogg N: Calpain 2 controls turnover of LFA-1 adhesions on migrating T lymphocytes. PLoS One 5: e15090, 2010.

26. Hussain AM, Zhang QX and Murray AG: Endothelial cell calpain activity facilitates lymphocyte diapedesis. Am J Transplant 5: 2640-2648, 2005.

27. Mikosik A, Foerster J, Jasiulewicz A, Frąckowiak J, Colonna-Romano G, Bulati M, Buffa S, Martorana A, Caruso C, Bryl E, et al: Expression of calpain-calpastatin system (CCS) member proteins in human lymphocytes of young and elderly individuals; pilot baseline data for the CALPACENT project. Immun Ageing 10: 27, 2013.

28. Kobayashi Y, Yamamoto K, Saido T, Kawasaki H, Oppenheim JJ and Matsushima K: Identification of calcium-activated neutral protease as a processing enzyme of human interleukin 1 alpha. Proc Natl Acad Sci USA 87: 5548-5552, 1990.

29. Zheng Y,Humphry M, Maguire JJ, Bennett MR and Clarke MCH: Intracellular interleukin-1 receptor 2 binding prevents cleavage and activity of interleukin-1 $\alpha$, controlling necrosis-induced sterile inflammation. Immunity 38: 285-295, 2013

30. McCarthy DA, Ranganathan A, Subbaram S, Flaherty NL, Patel N, Trebak M, Hempel N and Melendez JA: Redox-control of the alarmin, Interleukin-1 $\alpha$. Redox Biol 1: 218-225, 2013.

31. Pan HC, Yang CN, Hung YW, Lee WJ, Tien HR, Shen CC, Sheehan J, Chou CT and Sheu ML: Reciprocal modulation of $\mathrm{C} / \mathrm{EBP}-\alpha$ and $\mathrm{C} / \mathrm{EBP}-\beta$ by IL-13 in activated microglia prevents neuronal death. Eur J Immunol 43: 2854-2865, 2013.

32. Han Y, Weinman S, Boldogh I, Walker RK and Brasier AR Tumor necrosis factor-alpha-inducible IkappaBalpha proteolysis mediated by cytosolic m-calpain. A mechanism parallel to the ubiquitin-proteasome pathway for nuclear factor-kappab activation. J Biol Chem 274: 787-794, 1999.
33. Averna M, Stifanese R, De Tullio R, Salamino F, Bertuccio M, Pontremoli $S$ and Melloni E: Proteolytic degradation of nitric oxide synthase isoforms by calpain is modulated by the expression levels of HSP90. FEBS J 274: 6116-6127, 2007.

34. Bellocq A, Doublier S, Suberville S, Perez J, Escoubet B, Fouqueray B, Puyol DR and Baud L: Somatostatin increases glucocorticoid binding and signaling in macrophages by blocking the calpain-specific cleavage of Hsp 90. J Biol Chem 274: 36891-36896, 1999.

35. Li X, Luo R, Jiang R, Meng X, Wu X, Zhang S and Hua W: The role of the Hsp90/Akt pathway in myocardial calpain-induced caspase-3 activation and apoptosis during sepsis. BMC Cardiovasc Disord 13: 8, 2013.

36. Stalker TJ, Gong Y and Scalia R: The calcium-dependent protease calpain causes endothelial dysfunction in type 2 diabetes. Diabetes 54: 1132-1140, 2005.

37. Letavernier E, Perez J, Bellocq A, Mesnard L, de Castro Keller A, Haymann JP and Baud L: Targeting the calpain/calpastatin system as a new strategy to prevent cardiovascular remodeling in angiotensin II-induced hypertension. Circ Res 102: 720-728, 2008.

38. Zhao Y, Malinin NL, Meller J, Ma Y, West XZ, Bledzka K, Qin J, Podrez EA and Byzova TV: Regulation of cell adhesion and migration by Kindlin-3 cleavage by calpain. J Biol Chem 287: 40012-40020, 2012.

39. Franco SJ and Huttenlocher A: Regulating cell migration: Calpains make the cut. J Cell Sci 118: 3829-3838, 2005.

40. Cortesio CL, Boateng LR, Piazza TM, Bennin DA and Huttenlocher A: Calpain-mediated proteolysis of paxillin negatively regulates focal adhesion dynamics and cell migration. J Biol Chem 286: 9998-10006, 2011.

41. Cui Z, Han Z, Li Z, Hu H, Patel JM, Antony V, Block ER and Su Y: Involvement of calpain-calpastatin in cigarette smoke-induced inhibition of lung endothelial nitric oxide synthase. Am J Respir Cell Mol Biol 33: 513-520, 2005

42. Dong Y, Wu Y, Wu M, Wang S, Zhang J, Xie Z, Xu J, Song P, Wilson K, Zhao Z, et al: Activation of protease calpain by oxidized and glycated LDL increases the degradation of endothelial nitric oxide synthase. J Cell Mol Med 13 (no. 9A): 2899-2910, 2009.

43. Wang S, Peng Q, Zhang J and Liu L: $\mathrm{Na}^{+} / \mathrm{H}^{+}$exchanger is required for hyperglycaemia-induced endothelial dysfunction via calcium-dependent calpain. Cardiovasc Res 80: 255-262, 2008.

44. Nathan C and Ding A: Nonresolving inflammation. Cell 140: 871-882, 2010.

45. Bergounioux J, Elisee R, Prunier A-L, Donnadieu F, Sperandio B, Sansonetti P and Arbibe L: Calpain activation by the Shigella flexneri effector VirA regulates key steps in the formation and life of the bacterium's epithelial niche. Cell Host Microbe 11: 240-252, 2012

46. Smani Y, Docobo-Pérez F, McConnell MJ and Pachón J: Acinetobacter baumannii-induced lung cell death: Role of inflammation, oxidative stress and cytosolic calcium. Microb Pathog 50: 224-232, 2011.

47. Li M, Wang X, Yu Y, Yu Y, Xie Y, Zou Y, Ge J, Peng T and Chen R: Coxsackievirus B3-induced calpain activation facilitates the progeny virus replication via a likely mechanism related with both autophagy enhancement and apoptosis inhibition in the early phase of infection: An in vitro study in $\mathrm{H} 9 \mathrm{c} 2$ cells. Virus Res 179: 177-186, 2014.

48. Liu D, Yan Z, Minshall RD, Schwartz DE, Chen Y and Hu G: Activation of calpains mediates early lung neutrophilic inflammation in ventilator-induced lung injury. Am J Physiol Lung Cell Mol Physiol 302: L370-L379, 2012.

49. Celes MRN, Malvestio LM, Suadicani SO, Prado CM, Figueiredo MJ, Campos EC, Freitas ACS, Spray DC, Tanowitz HB, da Silva JS, et al: Disruption of calcium homeostasis in cardiomyocytes underlies cardiac structural and functional changes in severe sepsis. PLoS One 8: e68809, 2013.

50. Li X, Li Y, Shan L, Shen E, Chen R and Peng T: Over-expression of calpastatin inhibits calpain activation and attenuates myocardial dysfunction during endotoxaemia. Cardiovasc Res 83: 72-79, 2009.

51. Smith IJ, Lecker SH and Hasselgren P-O: Calpain activity and muscle wasting in sepsis. Am J Physiol Endocrinol Metab 295: E762-E771, 2008.

52. Hu H, Li X, Li Y, Wang L, Mehta S, Feng Q, Chen R and Peng T: Calpain-1 induces apoptosis in pulmonary microvascular endothelial cells under septic conditions. Microvasc Res 78: 33-39, 2009. 
53. Supinski GS, Wang W and Callahan LA: Caspase and calpain activation both contribute to sepsis-induced diaphragmatic weakness. J Appl Physiol 107, 1389-1396, 2009.

54. Wei W, Fareed MU, Evenson A, Menconi MJ, Yang H, Petkova V and Hasselgren PO: Sepsis stimulates calpain activity in skeleta muscle by decreasing calpastatin activity but does not activate caspase-3. Am J Physiol Regul Integr Comp Physiol 288: R580-R590, 2005.

55. Kraemer BF, Campbell RA, Schwertz H, Franks ZG, Vieira de Abreu A, Grundler K, Kile BT, Dhakal BK, Rondina MT, Kahr WHA, et al: Bacteria differentially induce degradation of Bcl-xL, a survival protein, by human platelets. Blood 120: 5014-5020, 2012.

56. Zafrani L, Gerotziafas G, Byrnes C, Hu X, Perez J, Lévi C, Placier S, Letavernier E, Leelahavanichkul A, Haymann JP, et al: Calpastatin controls polymicrobial sepsis by limiting procoagulant microparticle release. Am J Respir Crit Care Med 185: 744-755, 2012.

57. Kumar V, Everingham S, Hall C, Greer PA and Craig AWB: Calpains promote neutrophil recruitment and bacterial clearance in an acute bacterial peritonitis model. Eur J Immunol 44: 831-841, 2014

58. Samantaray S, Knaryan VH, Shields DC and Banik NL: Critical role of calpain in spinal cord degeneration in Parkinson's disease. J Neurochem 127: 880-890, 2013.

59. Smolock AR, Mishra G, Eguchi K, Eguchi S and Scalia R: Protein kinase $\mathrm{C}$ upregulates intercellular adhesion molecule-1 and leukocyte-endothelium interactions in hyperglycemia via activation of endothelial expressed calpain. Arterioscler Thromb Vasc Biol 31: 289-296, 2011.

60. Loot AE, Pierson I, Syzonenko T, Elgheznawy A, Randriamboavonjy V, Zivković A, Stark $\mathrm{H}$ and Fleming I: $\mathrm{Ca}^{2+}$-sensing receptor cleavage by calpain partially accounts for altered vascular reactivity in mice fed a high-fat diet. J Cardiovasc Pharmacol 61: 528-535, 2013.

61. Chen B, Zhao Q, Ni R, Tang F, Shan L, Cepinskas I, Cepinskas G, Wang W, Schiller PW and Peng T: Inhibition of calpain reduces oxidative stress and attenuates endothelial dysfunction in diabetes. Cardiovasc Diabetol 13: 88, 2014.

62. Li Y, Arnold JMO, Pampillo M, Babwah AV and Peng T: Taurine prevents cardiomyocyte death by inhibiting NADPH oxidase-mediated calpain activation. Free Radic Biol Med 46 $51-61,2009$.
63. Shan L, Li J, Wei M, Ma J, Wan L, Zhu W, Li Y, Zhu H, Arnold JMO and Peng T: Disruption of Racl signaling reduces ischemia-reperfusion injury in the diabetic heart by inhibiting calpain. Free Radic Biol Med 49: 1804-1814, 2010.

64. Li Y, Li Y, Feng Q, Arnold M and Peng T: Calpain activation contributes to hyperglycaemia-induced apoptosis in cardiomyocytes. Cardiovasc Res 84: 100-110, 2009.

65. Scalia R, Gong Y, Berzins B, Freund B, Feather D, Landesberg G and Mishra G: A novel role for calpain in the endothelial dysfunction induced by activation of angiotensin II type 1 receptor signaling. Circ Res 108: 1102-1111, 2011.

66. Ma J, Wei M, Wang Q, Li J, Wang H, Liu W, Lacefield JC, Greer PA, Karmazyn M,Fan GC, et al: Deficiency of Capn4 gene inhibits nuclear factor- $\kappa \mathrm{B}(\mathrm{NF}-\kappa \mathrm{B})$ protein signaling/inflammation and reduces remodeling after myocardial infarction. J Biol Chem 287: 27480-27489, 2012.

67. Li Y, Ma J, Zhu H, Singh M, Hill D, Greer PA, Arnold JM, Abel ED and Peng T: Targeted inhibition of calpain reduces myocardial hypertrophy and fibrosis in mouse models of type 1 diabetes. Diabetes 60: 2985-2994, 2011.

68. Aich J, Mabalirajan U, Ahmad T, Agrawal A and Ghosh B: Loss-of-function of inositol polyphosphate-4-phosphatase reversibly increases the severity of allergic airway inflammation. Nat Commun 3: 877, 2012.

69. Morita M, Banno Y, Dohjima T, Nozawa S, Fushimi K, Fan DG, Ohno T, Miyazawa K, Liu N and Shimizu K: Mu-calpain is involved in the regulation of TNF-alpha-induced matrix metalloproteinase-3 release in a rheumatoid synovial cell line. Biochem Biophys Res Commun 343: 937-942, 2006.

70. Chakrabarti S, Rizvi M, Morin K, Garg R and Freedman JE: The role of CD40L and VEGF in the modulation of angiogenesis and inflammation. Vascul Pharmacol 53: 130-137, 2010.

71. Wang Y, Zheng D, Wei M, Ma J, Yu Y, Chen R, Lacefield JC, $\mathrm{Xu} \mathrm{H}$ and Peng T: Over-expression of calpastatin aggravates cardiotoxicity induced by doxorubicin. Cardiovasc Res 98: 381-390, 2013. 